\title{
L'écrit diplomatique à Saint-Victor de Marseille et en Provence (ca. 950-ca. 1120)
}

Thèse de doctorat en histoire, sous la direction de Benoit-Michel Tock, université de Strasbourg, soutenue le 13 septembre 2013.

Jean-Baptiste Renault

\section{(2) OpenEdition}

Journals

Édition électronique

URL : https://journals.openedition.org/cem/13392

DOI : $10.4000 /$ cem. 13392

ISSN : 1954-3093

Éditeur

Centre d'études médiévales Saint-Germain d'Auxerre

Référence électronique

Jean-Baptiste Renault, «L'écrit diplomatique à Saint-Victor de Marseille et en Provence (ca. 950-ca.

1120) », Bulletin du centre d'études médiévales d'Auxerre / BUCEMA [En ligne], 18.1 | 2014, mis en ligne le

18 juin 2014, consulté le 22 septembre 2022. URL : http://journals.openedition.org/cem/13392 ; DOI https://doi.org/10.4000/cem.13392

Ce document a été généré automatiquement le 22 septembre 2022.

\section{c) (i) (8)(2)}

Creative Commons - Attribution - Pas d'Utilisation Commerciale - Partage dans les Mêmes Conditions 4.0 International - CC BY-NC-SA 4.0

https://creativecommons.org/licenses/by-nc-sa/4.0/ 


\section{L'écrit diplomatique à Saint-Victor de Marseille et en Provence (ca. 950-} ca. 1120)

Thèse de doctorat en histoire, sous la direction de Benoit-Michel Tock, université de Strasbourg, soutenue le 13 septembre 2013.

\section{Jean-Baptiste Renault}

1 À l'origine des travaux dont il est ici question se trouve la question de l'existence de «régions diplomatiques » (Urkundenlandschaften), que l'on pourrait définir comme des espaces marqués par

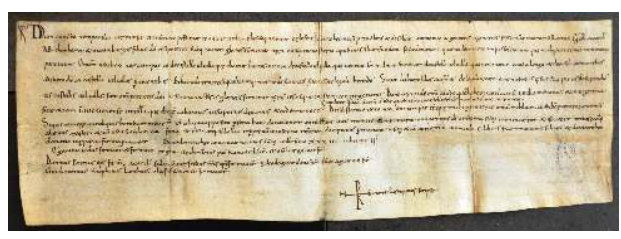
des pratiques communes dans l'écrit diplomatique ${ }^{1}$. Des disparités régionales dans les pratiques de l'écrit à l'époque carolingienne ont été mises en évidence. C'est pourquoi, il semble intéressant de savoir si dans le processus de territorialisation qui a suivi l'effritement de l'empire carolingien, des espaces se sont individualisés culturellement par leurs pratiques documentaires ${ }^{2}$. Par ailleurs, les $\mathrm{x}^{\mathrm{e}}$ et $\mathrm{xl}^{\mathrm{e}}$ siècles sont connus pour leurs profondes mutations dans le domaine de l'écrit diplomatique. Celles-ci ont affecté à la fois la production de l'écrit (élément humain), la forme des actes et leur typologie, mais aussi leur conservation ${ }^{3}$. Une " prise de pouvoir monastique » dans le domaine de l'écrit a été évoquée par Olivier Guyotjeannin ${ }^{4}$. Le lien entre ces changements dans l'écrit et l'évolution de la société reste cependant à approfondir. En évaluant l'hypothèse de l'existence d'une région diplomatique, l'enquête a davantage fait émerger la notion de centres d'écritures, entendus comme des institutions contrôlant et conservant l'écrit. Les modalités de cet investissement nouveau du terrain offert par la diplomatique ainsi que ses conséquences ont pu être mises en relation avec l'histoire particulière des monastères.

2 La richesse des sources provençales a permis de mener une enquête régionale, avec des regards sur les régions voisines et sur une sélection d'institutions non-provençales bien 
documentées. En outre, une riche historiographie récente relative aux rapports entre l'aristocratie et l'Église au temps des réformes ecclésiales a facilité la contextualisation ${ }^{5}$. Héritière d'un royaume carolingien absorbé dans le royaume de Bourgogne $^{6}$, la Provence demeure aux $\mathrm{x}^{\mathrm{e}}$ et $\mathrm{xI}^{\mathrm{e}}$ siècles une réalité tangible car la Provincia et les provinciales apparaissent dans les sources. C'est précisément ce cadre ecclésiastique des trois provinces d'Arles, d'Aix-en-Provence et d'Embrun qui a été choisi pour cette enquête. Le renouveau de la Provence, après la période troublée du premier $\mathrm{x}^{\mathrm{e}}$ siècle, constitue le point de départ chronologique. Son terme, vers 1120 , a permis d'intégrer une période qui a transmis moins d'actes (ca. 1080-ca. 1120), mais qui, en revanche, a connu une vague de rédaction de cartulaires par lesquels une part considérable d'actes antérieurs a été transmise. La Provence offre, de fait, un corpus documentaire riche et typologiquement varié. Il s'agit d'une des régions françaises les plus favorisées, d'une part, pour la conservation des originaux, d'autre part, grâce à une série de cartulaires monastiques et cathédraux qui a transmis un nombre d'actes considérable, soit environ 1500 entre 941 et 1120, dont un peu plus de la moitié pour Saint-Victor de Marseille (857) ${ }^{7}$.

3 Avant d'étudier la production de l'écrit à travers son élément humain et les phénomènes de la rédaction, il fallait soupeser les sources et leur représentativité en interrogeant les pratiques archivistiques et la transmission des actes. L'enquête a été plus approfondie pour Saint-Victor de Marseille du fait de l'ampleur de son fonds et de la grande diversité typologique des documents transmis. À partir d'un examen matériel des actes il a été possible de proposer quelques hypothèses sur le travail archivistique des Victorins. Les premiers efforts archivistiques ne sont tangibles que dans la période 1060-1079 à travers certaines vagues d'annotation, de classement et probablement un rassemblement à Marseille d'actes antérieurement conservés dans les dépendances notamment par le recours à des pancartes relatives à une dépendance. Entre 1060 et 1079, un groupe d'actes s'est distingué par un traitement spécial: il s'agit des documents pontificaux, des lettres ainsi que des actes concernant les dépendances situées à l'ouest du Rhône, qui, conservés à part, auraient pu constituer un dossier sous le contrôle direct de l'abbé. L'abbatiat de Durand (1060-1065) apparaît désormais comme un tournant dans l'intérêt pour les documents et leur organisation. L'attention nouvelle aux archives, apparue après 1060, a favorisé en amont le projet de rédaction du cartulaire, projet éclos à la fin de l'abbatiat de Bernard. Le grand cartulaire de SaintVictor de Marseille, composé en 1079, a apporté des renseignements nombreux sur le classement et la pratique des archives, car sa structure reflète certainement celle du chartrier au moment de la rédaction ${ }^{8}$. Lié à la composition d'une liste de biens confirmée par Grégoire VII, le cartulaire est le fruit d'une décision de l'abbé Bernard (1065-1079), qui aurait pu suivre l'exemple de Cluny, abbaye où il avait séjourné peu avant le lancement de la compilation ${ }^{9}$. Commencé par Jean de Ripoll, moine de SaintVictor et scribe de chartes, le travail de compilation a été une œuvre collective animée par quelques principes de transcriptions et une méthode élaborée progressivement dans un souci de conformité chez la plupart des scribes, tandis que le dernier tiers du recueil se distingue ${ }^{10}$. Recueil domanial, le cartulaire s'organise de façon topographique, des sections diocésaines regroupant des dossiers par lieux ou dépendances, dont les contours, la structure interne et le signalement ont pu être affinés en cours de compilation. Malgré l'absence des privilèges de Grégoire VII au cartulaire, le dossier introductif affirmait le statut particulier du monastère et comportait des signes de l'affirmation des abbés dans la Réforme grégorienne. 
4 Si ces pratiques permettent d'évoquer une véritable politique archivistique, les objectifs de celle-ci devaient être mieux connus. Une vive sensibilité au rôle des actes s'est exprimée par une utilisation dans le cadre de la reconquête du patrimoine, lancée par l'abbé Isarn (1021-1047), mais aussi par une réception attentive de l'écrit exogène dans le grand cartulaire. Les cartularistes ont nettement privilégié les actes des églises héritées et des monastères rattachés par rapport aux actes pour des laïcs ${ }^{11}$. De ce fait, le cartulaire a valorisé le mouvement de transfert de sanctuaires, en accordant une place privilégiée aux actes de transfert d'églises, qui, le plus souvent, étaient copiés en tête des dossiers des églises rattachées. Pareillement, les actes de consécrations ou de dotations d'églises ont été signalés par des rubriques renseignant leur nature juridique (sponsalicium). Ainsi, le cartulaire non seulement valorisait le mouvement de liberté des églises et leur arrivée dans le giron marseillais, mais encore relayait les discours ecclésiologiques développés dans les chartes de consécrations, principalement dans les années 1050. Toutefois, si la mémoire des petites églises a été facilement absorbée par l'ensemble victorin, l'attitude envers les monastères non provençaux rattachés a été plus complexe. Mais, dépassant son rayonnement provençal, le monastère avait assumé une vocation plus large de tête d'une congrégation méditerranéenne et d'allié de la papauté - en Catalogne ou en Sardaigne particulièrement ${ }^{12}$. L'édification progressive d'une véritable congrégation marseillaise, soumise à "l'abbé de Marseille " - ainsi qualifié dans les actes -, s'est accompagnée de réalisations de copies et parfois de prélèvement dans les archives des monastères languedociens et catalans rattachés. Ces effets concrets sur la gestion et le contrôle des archives confirment la sensibilité aiguë observée chez les moines marseillais pour la valeur de l'acte en tant qu'outil de communication.

5 Si l'histoire de Saint-Victor et le positionnement de ses abbés dans la Réforme de l'Église explique en partie les options de sa politique archivistique, il apparaît par comparaison que les autres monastères et chapitres provençaux n'ont pas connu une prise de conscience aussi précoce. Ainsi, à Lérins, la rédaction d'un cartulaire est plus tardive et s'est déroulée en deux étapes, d'abord une compilation limitée, dont nous avons pu montrer qu'elle était en partie liée à l'obtention d'un privilège pontifical, puis une compilation plus systématique ${ }^{13}$. À Montmajour, on trouve des signes d'une décentralisation des documents, comme l'indiquent certains groupes de notes dorsales et l'examen du cartulaire du prieuré de Correns, réalisé au xII ${ }^{e}$ siècle. On ne peut parler pour Montmajour d'une véritable politique archivistique. L'histoire propre des monastères l'emportant sur les similitudes dans les pratiques de conservation, il n'y a pas de « région archivistique ». Toutefois, on pourra signaler qu'une certaine émulation a pu animer les chapitres cathédraux dans la rédaction de cartulaires, la chronologie pouvant être interprétée comme une tache d'huile à partir d'Arles, à la fin du XI siècle et au début du XII siècle. L'approche de la conservation et de la transmission - des regards sont portés en annexe sur les canaux et les biais modernes de celle-ci - a permis d'aborder la représentativité des sources - sans pouvoir donner beaucoup d'assurances sur celle-ci - avant de passer à l'étude de la production de l'écrit. 


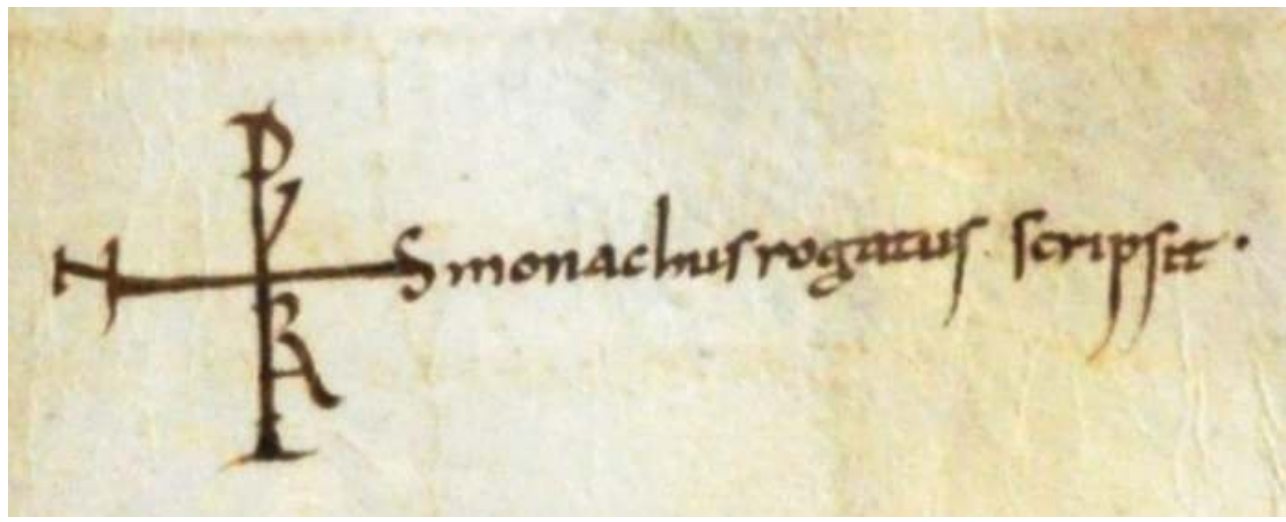

6 C'est tout d'abord à travers les scribes que l'on peut approcher la production des actes. La richesse des souscriptions de scribes transmises par les actes originaux et parfois par les cartulaires a permis d'esquisser une prosopographie des scribes provençaux ${ }^{14}$. Parmi les 230 scribes identifiés, les mieux attestés ont pu faire l'objet d'une étude plus fine qui a permis d'interroger leur implication dans la rédaction - le scribe ne fait-il qu'écrire ou compose-t-il réellement le texte ? -, leur rapport aux bénéficiaires, leurs " clients ", leur inscription dans un cadre géographique et institutionnel, et enfin leur carrière et leur itinéraire social. Deux grandes périodes sont apparues. Tout d'abord, entre le milieu du $\mathrm{x}^{\mathrm{e}}$ siècle et les premières années $\mathrm{du} \mathrm{XI}^{\mathrm{e}}$ siècle, les scribes sont majoritairement actifs dans les cités épiscopales - la plupart des mentions concernant Arles, Avignon et Apt - et ils écrivent pour une clientèle multiple ${ }^{15}$. Ces scribes, le plus souvent des prêtres, prolongeaient en quelque sorte la tradition du notariat carolingien. Ils écrivaient aussi bien pour des bénéficiaires ecclésiastiques que pour des laïcs, seuls les moines semblant alors réserver leur production aux églises. Ces scribes, à la clientèle multiple, disparaissent lors du premier tiers $d u \mathrm{XI}^{\mathrm{e}}$ siècle avec tout un pan de la diplomatique - actes de précaires, d'échanges et ventes. Au début du XI ${ }^{e}$ siècle, en effet, les scribes écrivent majoritairement pour l'institution à laquelle ils appartiennent et ne se mettent plus au service d'autres bénéficiaires. Pour certains d'entre eux, il a été possible de retracer une carrière sur plusieurs décennies, principalement pour des moines de Saint-Victor. L'accaparement de l'écriture par les bénéficiaires a pour conséquence une diversification des lieux de l'écriture : les moines ont été plus enclins à circuler à travers la Provence pour recevoir des aumônes et dresser des actes, comme cela a été identifié pour des scribes victorins, qui indiquent dans leur souscription avoir été missionnés par leur abbé.

7 L'étude de la rédaction a permis non seulement d'affiner la connaissance de l'organisation de l'écrit, mais encore d'évaluer l'implication des institutions et, au-delà, leur conception du rôle de l'écrit documentaire. L'enquête a privilégié les parties introductives du discours, invocation, préambule et adresse. Avant d'aborder l'empreinte des institutions sur l'écrit documentaire, une approche typologique de la "vie des formes» diplomatiques a été proposée en analysant les circulations, les emprunts au chartrier, les créations.

8 Si le milieu du $\mathrm{x}^{\mathrm{e}}$ siècle a connu un réinvestissement des pratiques documentaires, celui-ci s'est abreuvé à plusieurs sources : des modèles locaux, des modèles venus de 
l'Ouest, mais aussi des modèles francs, probablement conservés par le truchement de recueils. Autour de l'Église d'Arles, un réseau s'est formé par les itinéraires des clercs. Cela expliquerait les circulations de formules en Provence occidentale jusqu'au début $\mathrm{du} \mathrm{XI} \mathrm{e}^{\mathrm{e}}$ siècle. Ce réseau est à l'origine d'un patrimoine commun à la région constitué par certaines formules.

9 Toutefois, l'empreinte de l'institution bénéficiaire n'a pas tardé à infléchir le formulaire des actes. Dès la fin du $\mathrm{x}^{\mathrm{e}}$ siècle, des scribes monastiques ou d'anciens moines ont acclimaté et modifié le formulaire traditionnel. À Montmajour, le cadre formel est assez rigide, mais laisse dans les interstices du formulaire s'épanouir des formules valorisant l'aumône et le rôle de saint Pierre, patron du monastère. C'est toutefois à Saint-Victor que l'indépendance vis-à-vis de la tradition est la plus nette et, partant, que la « saveur propre» (Olivier Guyotjeannin) est la plus affirmée. Au début du XI ${ }^{e}$ siècle, se sont développées des formes ornées à travers les préambules, dont la grande variété est imputable à une multiplicité des scripteurs. Si les actes victorins ont une coloration particulière, des formes plus localisées sont apparues du fait d'une décentralisation de l'écrit. À travers préambules religieux et exposés valorisant l'aumône, les Victorins ont développé une pastorale en direction des laïcs dans un contexte d'essor et de reconquête du patrimoine ancien ${ }^{16}$. À Lérins, enfin, pour parer à une tradition défaillante, un répertoire composite s'est constitué, formé d'emprunts à quelques rares modèles anciens conservés dans le chartrier, de formules chinées dans les actes d'églises voisines et de quelques compositions nouvelles. Il s'agissait de reconstituer un patrimoine formel faisant défaut à partir de sources variées, par des innovations et de nouvelles combinaisons.

10 L'histoire des institutions religieuses a permis de mieux comprendre les inflexions du formulaire, bien que monastères et chapitres n'aient pas connu la même implication dans la rédaction. La capacité de Saint-Victor à modifier profondément le « profil » des actes est apparue à travers deux phénomènes : tout d'abord l'efflorescence de formes originales entre 1005 et 1060, puis l'abandon brutal de ces formes au profit d'un formulaire simplifié. Alors que les rédactions victorines, jusque vers le milieu du $\mathrm{XI}^{\mathrm{e}}$ siècle, valorisaient l'aumône des laïcs, elles ont été concurrencées, dès la fin des années 1040, par des compositions plus ecclésiologiques, systématisant une valorisation de la restitution des biens d'Église en des mains ecclésiastiques. Cela correspondait à un mouvement de transferts de sanctuaires et de consécrations ayant culminé à la fin de l'abbatiat de Pierre (1047-1060) ${ }^{17}$. L'élection de l'abbé Durand (1060-1065) serait responsable de profondes mutations: d'une part, on abandonna en Provence le préambule au profit d'invocations et de formes plus simples, d'autre part, pour une série d'actes de rattachement de monastères situés à l'Ouest du Rhône, des préambules teintés d'une ecclésiologie radicalisée vinrent justifier l'action des Marseillais.

11 Ces évolutions successives, qui ailleurs ne sont jamais aussi marquées, indiquent une forte sensibilité victorine à la valeur de média de l'acte et aux possibilités qu'il offre. Dans la phase pastorale, la piété des donateurs est particulièrement mise en scène. Un premier tournant du rôle de l'acte pourrait apparaître avec les thèmes ecclésiologiques développés dans les chartes de consécrations. Mais c'est après 1060 que le discours est réorienté des laïcs vers les seuls moines. Ce changement constituait une forme de radicalisation montrant à quel point les abbés Durand et Bernard étaient engagés dans une réforme affirmant la séparation des sphères laïque et ecclésiastique. 
12 L'affirmation des identités dans les actes a permis de nuancer ce tableau. Cette affirmation s'est exprimée particulièrement dans ce que nous avons appelé les "formules de désignation", c'est-à-dire l'équivalent, pour une institution, de la titulature d'un particulier. Pouvant demeurer figées, ces formules sont parfois mouvantes et ouvertes à certaines mises en valeur des saints patrons. C'est le cas avec l'insistance sur le rôle d'intercesseur de saint Pierre à Montmajour, de la fin du $\mathrm{x}^{\mathrm{e}}$ siècle à 1030 environ. Elle s'inscrit dans un schéma classique du don et du contre-don, le monastère pétrinien s'associant au rôle de portier de l'au-delà de son saint patron. À Arles, la figure de saint Trophime, mise en valeur dès la fin du $x^{e}$ siècle, a été utilisée plus encore sous l'archevêque Raimbaud de Reillane, affirmant dans ses chartes l'apostolicité de l'Église d'Arles en s'appuyant sur la lettre du pape Zosime ${ }^{18}$. Cette attention nouvelle de la part de Raimbaud, ancien moine de Saint-Victor, apparaissait comme un parallèle aux efforts identitaires du monastère marseillais. En effet, dans la première moitié $\mathrm{du} \mathrm{XI}$ siècle, le martyr Victor et sa sépulture ont fait l'objet d'une mise en valeur croissante, parallèlement à un travail de synthèse hagiographique ${ }^{19}$. Après 1060, sans disparaître des chartes et de leurs formules dévotionnelles, Victor se voit alors nettement concurrencé par Jean Cassien. Le rôle de Cassien dans la naissance du monachisme en Gaule se voit nettement revendiqué dans les chartes, mais aussi dans la Vie d'Isarn, proposant un nouveau modèle de sainteté monastique, au détriment du saint militaire Victor, auquel la famille des vicomtes de Marseille demeurait très attachée ${ }^{20}$. Cette valorisation nouvelle s'est exprimée le plus souvent dans des actes liés à l'édification d'une congrégation marseillaise et a été mise au service de discours d'auto-légitimation. Ainsi, à travers l'analyse des valeurs d'usages identitaires ou mémorielles des chartes, il a été possible de saisir des périodes où elles ont fait l'objet d'un investissement particulier au service d'une politique, qu'il s'agisse des formules valorisant l'aumône au saint patron ou des figures tutélaires exhumées dans une optique précise : Trophime, pour affirmer la prééminence du siège arlésien, ou Cassien, pour légitimer le pouvoir du monastère marseillais sur les abbayes rattachées. Les premiers siècles chrétiens ont constitué un passé utile pour les hommes d'Église à partir du deuxième quart du XI siècle.

13 La question initiale de l'existence d'une "région diplomatique " s'est vue finalement dépassée. Un répertoire formel régional a bien existé en Provence au $x^{e}$ siècle, grâce à la circulation des clercs au $\mathrm{x}^{\mathrm{e}}$ siècle - le plus souvent à partir de la cité d'Arles -, sans disparaître au siècle suivant. Cependant, le fait dominant au XI $\mathrm{x}^{\mathrm{e}}$ siècle, est davantage l'affirmation des établissements dans le domaine de l'écriture documentaire, composant ainsi un paysage plus contrasté. Fortement liés à l'aristocratie, les monastères n'ont pas abordé de la même façon la diplomatique. Saint-Victor de Marseille a connu le parcours le plus riche en étapes bien marquées, pouvant souvent être mises en relation avec l'histoire du monastère et l'engagement de ses abbés dans la Réforme de l'Église. Cela s'est exprimé par un volontarisme des abbés et de certains moines-scribes, qui s'est manifesté par une aptitude particulière à infléchir le formulaire des actes. En outre, un souci précoce des archives, né sous l'abbatiat d'Isarn, accru sous l'abbé Durand, a abouti à la rédaction du grand cartulaire, à la fin de l'abbatiat de Bernard. L'implication des autres monastères dans la rédaction des actes et leur utilisation a posteriori semble avoir été plus tiède et l'attention aux archives y a été plus tardive. En définitive, outre une chronologie marquée par deux grandes ruptures, c'est une appréciation différente du rôle de l'écrit documentaire selon les 
institutions qui a pu être approchée. Seul Saint-Victor a pris toute la mesure, pour mener sa politique ecclésiale, des possibilités offertes par l'acte écrit, en tant qu'outil de communication et de contrôle social.

Reçu : 13 mars 2014 - Accepté : 27 mai 2014

\section{NOTES}

1. La question a surtout été lancée par H. FICHTENAU, Das Urkundenwesen in Österreich vom 8. bis zum frühen 13. Jahrhundert, Vienne, 1971.

2. Le concept de "régions diplomatiques" avant l'an Mil a fait l'objet d'une monographie pour la Rhétie, dont les pratiques diffèrent fortement de celle des régions voisines (P. ERHART et J. KLEINDINST, Urkundenlandschaft Rätien, Vienne, 2004) et du colloque de Saint-Gall en 2006 (P. ERHART, K. HEIDECKER et B. ZELLER (dir.), Die Privaturkunden der Karolingerzeit, Zurich, 2009). Sur le processus de territorialisation, voir A. BRENDLER, "Territorialisation : un concept de l'historiographie allemande ", in Territorium: Raum und Politik: Wahrnehmung und Praxis im Frankenreich und seinen Nacholgereichen vom 9. Bis zum 13. Jahrhundert [http://tobias-lib.uni-tuebingen.de/ volltexte/2011/5965/pdf/Territorium_Brendler_url.pdf]; et pour la Provence : F. MAZEL, "Spatialisation et territorialisation des pouvoirs en Provence ( $\mathrm{x}^{\mathrm{e}}$-XII ${ }^{\mathrm{e}}$ siècle) : quelques éléments de réflexion ", in Territorium..., ibid. [http://tobias-lib.unituebingen.de/volltexte/2013/7136/pdf/Territorium_Mazel.pdf].

3. O. GUYOTJEANNin, «Penuria scriptorum. Le mythe de l'anarchie documentaire dans la France du Nord ( $\mathrm{x}^{\mathrm{e}}$ siècle-première moitié du XI $\mathrm{e}^{\mathrm{e}}$ siècle) $"$, in $\mathrm{O}$. GUYOTJEANNIN, L. MORELLE et M. PARISSE (éd.), Pratiques de l'écrit documentaire au XI siècle, BEC, 155 (1997), p. 11-44. D. BARTHELEMY, «Une crise de l'écrit? Observations sur les actes de Saint-Aubin d'Angers ( $\mathrm{xl}^{\mathrm{e}}$ siècle) ", in O. GUYOTJEANNIN, L. MORELle et M. PARISSE (éd.), Pratiques..., ibid., p. 95-118. L. MORELLE, « Histoire et archives vers l'an Mil : une nouvelle «mutation »?», Histoire et archives, 3 (janvier-juin 1998), p. 119-141; ID., " Instrumentation et travail de l'acte : quelques réflexions sur l'écrit diplomatique en milieu monastique au $\mathrm{xl}^{\mathrm{e}}$ siècle ", Médiévales, 56 (printemps 2009), p. 41-74.

4. O. GUYotJEAnNIN, « Penuria scriptorum... », ibid.

5. La société féodale en Provence a fait l'objet de deux thèses : E. MAGNANI, Monastères et aristocratie en Provence milieu $X^{e}$-début XII siècle, Münster, 1999; F. MAZEL, La noblesse et l'Église en Provence fin $\mathrm{X}^{\mathrm{e}}$-début XIV siècle. L'exemple des familles d'Agoult-Simiane, de Baux et de Marseille, Paris, 2002. La chronologie des rapports aristocratie/Église a été précisée par F. MAZEL, «Amitié et rupture de l'amitié. Moines et grands laïcs provençaux au temps de la crise grégorienne (milieu XI ${ }^{\mathrm{e}}$-milieu XII ${ }^{\mathrm{e}}$ siècle) », Revue historique, 307 (2005), p. 53-95; en outre, les spécificités méridionales de la Réforme de l'Église ont été analysées lors du colloque de Fanjeaux, 9-12 juillet 2012, La Réforme "grégorienne » dans le Midi (milieu XI ${ }^{e}$-début XIII siècle), sous la direction de F. MAZEL, à paraître dans les Cahiers 
de Fanjeaux. Voir infra les références nombreuses relatives à Saint-Victor de Marseille au XI ${ }^{\mathrm{e}}$ siècle.

6. F. MAZEL, «La Provence entre deux horizons (843-1032). Réflexions sur un processus de régionalisation ", in M. GAILLARD et M. MARGUE (dir.), De la mer du Nord à la Méditerranée: Francia Media, une région au cceur de l'Europe c. 840-c. 1050, Luxembourg, 2011, p. 453-485.

7. Les originaux sont consultables sur la base de données: Chartes originales antérieures à 1121 conservées en France, éd. C. GIRAUD, J.-B. ReNAULT et B.-M. TOcK, Nancy (Centre de médiévistique Jean Schneider); éd. électronique, Orléans (Institut de recherche et d'histoire des textes), 2010 [http://www.cn-telma.fr/originaux/index/].

8. Le cartulaire était déjà bien connu par les études de M. ZERNER, notamment «L'élaboration du grand cartulaire de Saint-Victor de Marseille", in O. GUYotjeAnNIN, L. MORELle et M. PARISSE, Les cartulaires, Paris, 1993, p. 217-246 et «Le grand cartulaire de Saint-Victor de Marseille: comparaison avec Cluny, crise grégorienne et pratique d'écriture ", in M. FIXOT et J.-P. PELLETIER (éd.), Saint-Victor de Marseille, études archéologiques et historiques, Turnhout, 2009, p. 295-322.

9. D. IOGNA-PRAT, «La confection des cartulaires et l'historiographie à Cluny aux $\mathrm{XI}^{\mathrm{e}}$ XII ${ }^{\mathrm{e}}$ siècles ", in O. GUYOTJEANNIN, L. MORELLE et M. PARISSE, Les cartulaires..., ibid., p. 27-44. S. BARRET, La mémoire et l'écrit : l'abbaye de Cluny et ses archives (Xe-XVIII siècle), Münster, 2004, p. 108-109.

10. L'étude s'est appuyée sur un nouvel examen codicologique accompagné d'un repérage des mains - au nombre d'une quinzaine, sans compter les actes ajoutés après 1079-1080 - et d'une confrontation systématique entre les originaux et leur transcription au cartulaire.

11. Sur la sélection des actes transcrits, voir notre étude «Saint-Victor de Marseille, des archives au cartulaire : sélection des actes, contingences archivistiques et objectifs d'une compilation ( $\mathrm{XI}^{\mathrm{e}}$ siècle) ", in E. RAMIREZ-VAQUERO et V. LAMAZOU-DUPLAN (dir.), Los cartularios medievales. Escribir y conservar la memoria del poder, el poder de la memoria. Les cartulaires médiévaux. Écrire et conserver la mémoire du pouvoir, le pouvoir de la mémoire, Pau, 2013, p. 173-183.

12. E. MAGNANI, "Saint-Victor de Marseille, Cluny et la politique de Grégoire VII au nord-ouest de la Méditerranée ", in G. ConstABLE, G. Melville et J. OBERSTE (dir.), Die Cluniazenser in ihren politisch-sozialen Umfeld, Münster, 1998, p.321-347. L. voNES, «Päpstlicher Legat und päpstlicher Wille. $\mathrm{Zu}$ den Rahmenbedingungen der Legatengewalt um 1100 am Beispiel der Gesandtentätigkeit des Richard von Marseille », in S. WEINFURTER (dir.), Päpstliche Herrschaft im Mittelalter. Funktionsweisen-StrategienDarstellungsformen, Ostfildern, 2005, p. 335-360. V. POLONIO, «Il monasterio di S. Vittore di Marsiglia nell'alto Tirreno ", in F. ARNEODO et P. GUGLIELMOTTI (dir.), Attraverso le Alpi : S. Michele, Novalesa, S. Teofredo et altre reti monastiche, Bari, 2008, p. 223-243.

13. G. BUTAUD, "Listes abbatiales, chartes et cartulaire de Lérins: problèmes de chronologie et de datation (XI ${ }^{\mathrm{e}}$-XII ${ }^{\mathrm{e}}$ siècles) ", in Y. CODOU et M. LAUWERS (dir.), Lérins, une île sainte de l'Antiquité au Moyen Âge, Turnhout, 2009, p. 365-444.

14. Une série d'études récentes sur les scribes a permis de bien inscrire la Provence dans le paysage européen. B.-M. тоск, Scribes, souscripteurs et témoins dans les actes privés en France (VII -début XII siècle), Turnhout, 2005. M. ZIMMERMANN, Écrire et lire en Catalogne $d u$ IX $X^{e}$ au XII siècle, Madrid, 2003. J. BELMON, "In conscribendis donacionibus hic ordo servandus est. L'écriture des actes de la pratique en Languedoc et Toulousain ( $\mathrm{IX}^{\mathrm{e}}-\mathrm{x}^{\mathrm{e}}$ siècle) ", in M. ZIMMERMANN (dir.), Auctor et Auctoritas. Invention et conformisme dans 
l'écriture médiévale, Paris, 2001, p. 283-320, aux p.291-294. A. MEYER, Felix et inclitus notarius. Studien zum italienischen Notariat vom 7. bis zum 13. Jahrhundert, Tübingen, 2000. I. FEES, Eine Stadt lernt schreiben. Venedig vom 10. Bis zum 12. Jahrhundert, Tübingen, 2002.

15. J.-B. RENAULT, « Activity of Scribes and Life of Diplomatic Forms in Provence (10th century - early 11th century ", à paraître en 2014. À propos de l'écrit pour les laïcs, voir A. J. козто, « Laymen, Clerics and Documentary Practices in the Early Middle Ages : The Example of Catalonia ", Speculum, 80 (2005), p.44-74 ainsi que le volume récent W. BROWN, M. COSTAMBEYS, M. InNES et A. Kosto (éd.), Documentary Culture and the Laity in the Early Middle Ages, Cambridge, 2013.

16. E. MAGNANI, «Pastorale monastique et pratique de l'écrit. Les actes de donation de SaintVictor de Marseille (XI ${ }^{\mathrm{e}}$ siècle) », in M. FIXOT et J.-P. PELLETIER (éd.), Saint-Victor de Marseille..., op. cit., p. 239-254.

17. M. LAUWERS, «Consécration d'églises, réforme et ecclésiologie monastique. Recherches sur les chartes de consécration provençales du XI $\mathrm{x}^{\mathrm{e}}$ siècle », in $\mathrm{D}$. MÉHU (dir.), Mise en scène et mémoire de la consécration d'église dans l'Occident médiéval, Turnhout, 2007, p. 93-142.

18. La redécouverte de saint Trophime avait été observée par F. Mazel : « Fondements et horizons d'une hégémonie archiépiscopale. L'Église d'Arles d'Ithier (961-985) à Raimbaud (1030-1069)", in S. B ALOSSINO et G. B. G ARBARINO (éd.), L'organizzazione ecclesiastica nel tempo di san Guido. Istituzioni e territorio nel secolo XI, Acqui Terme, 2007, p. 105-138.

19. M. LAUWERS, «Cassien, le bienheureux Isarn et l'abbé Bernard, un moment charnière dans l'édification de l'église monastique provençale (1060-1080) », in M. FIXOT et J.-P. PELLETIER (éd.), Saint-Victor de Marseille..., op. cit., p. 213-238. Vie d'Isarn, abbé de Saint-Victor de Marseille (XI siècle), éd. C. CABY, J.-F. COTTIER, R. M. DESSI, M. LAUWERS, † J.-P. WEISS et M. ZERNER, Paris, 2010.

20. F. MAZEL, « De l'emprise aristocratique à l'indépendance monastique : patrimoine et culte des saints à Saint-Victor de Marseille », in M. FIXOT et J.-P. PELLETIER (éd.), SaintVictor de Marseille..., op. cit., p. 255-281.

\section{AUTEUR}

\section{JEAN-BAPTISTE RENAULT}

Ingénieur d'études, université de Lorraine 